\title{
EDGEWORTH EXPANSION ON $n$-SPHERES AND JACOBI HYPERGROUPS
}

\author{
Gyula Pap and Michael Voit
}

\begin{abstract}
Suitable normalisation of time-homogeneous rotation-invariant random walks on unit spheres $S^{d} \subset \mathbb{R}^{d+1}$ for $d \geqslant 2$ leads to a central limit theorem with a Gaussian limit measure. This paper is devoted to an associated Edgeworth expansion with respect to the total variation norm. This strong type of convergence is different from the classical case. The proof is performed in the more general setting of Jacobi-type hypergroups on an interval.
\end{abstract}

\section{INTRODUCTION}

The aim of the present paper is to derive an Edgeworth expansion for isotropic random walks on unit spheres $S^{d} \subset \mathbb{R}^{d+1}$ for $d \geqslant 2$ and, in general, on Jacobi hypergroups.

Let us consider a stationary random walk $\left(X_{n}\right)_{n \geqslant 0}$ on $S^{d}$ starting, say, at the North Pole $x_{0} \in S^{d}$ and having some $S O(d+1)$-invariant transition probability. The transition can be described by some probability measure $\mu \in M^{1}([0, \pi])$ which is the distribution of the angles

$$
\varangle\left(X_{n}, X_{n-1}\right) \in[0, \pi], \quad n \in \mathbb{N},
$$

between two successive jumps. For all $k \in \mathbb{N}$ let $\left(X_{n}^{(k)}\right)_{n \geqslant 0}$ be a shrinked isotropic random walk starting at $x_{0} \in S^{d}$ and associated with the probability measure $\mu_{k} \in$ $M^{1}([0, \pi / \sqrt{k}])$ defined by $\mu_{k}(A):=\mu(\sqrt{k} A)$ for Borel sets $A \subset[0, \pi / \sqrt{k}]$. This random walk is discussed, for instance, by Bingham [2] and by Voit [9]. From Voit [9], under a mild restriction on $\mu$ (' $\mu$ must not be concentrated in 0 too much'), there exists a $k_{0}=k_{0}(d, \mu)$ such that for each $k \geqslant k_{0}$ the distribution $\mu_{k}^{(k)}$ of $X_{k}^{(k)}$ has a continuous bounded density $f_{k}$ with respect to the uniform distribution $\omega_{d}$ on $S^{d}$. Moreover, it was shown in that paper that there is a 'Gaussian' measure $\nu_{0}^{\mu}$ on $S^{d}$ with a continuous bounded $\omega_{d}$-density $g_{0}$ such that

$$
\left\|f_{k}-g_{0}\right\|_{\infty}=O\left(k^{-1}\right), \quad\left\|\mu_{k}^{(k)}-\nu_{0}^{\mu}\right\|=O\left(k^{-1}\right) \quad \text { for } k \rightarrow \infty
$$

Received 23rd February, 1998

The paper was partially written while the first author was visiting the University of Tübingen, Germany, supported by the Alexander von Humboldt Foundation.

Copyright Clearance Centre, Inc. Serial-fee code: 0004-9729/98 \$A2.00+0.00. 
where $\|\varrho\|$ denotes the total variation norm of the measure $\varrho$, and the order $O\left(k^{-1}\right)$ is sharp.

In this paper we give bounded (signed) measures $\nu_{j}^{\mu}(j=1,2, \ldots)$ on $S^{d}$ with continuous bounded $\omega_{d}$-densities $g_{j}(j=1,2, \ldots)$ such that for all $s \in \mathbb{N}$

$$
\left\|f_{k}-\sum_{j=0}^{s-1} k^{-j} \cdot g_{j}\right\|_{\infty}=O\left(k^{-s}\right), \quad\left\|\mu_{k}^{(k)}-\sum_{j=0}^{s-1} k^{-j} \cdot \nu_{j}^{\mu}\right\|=O\left(k^{-s}\right) \quad \text { for } k \rightarrow \infty
$$

The expansion will be derived from a more general result valid for Jacobi hypergroups covering also the cases of compact symmetric spaces of rank one (which include the projective spaces; see, for instance, Helgason [7]).

\section{JACOBI HYPERGROUPS AND GAUSSIAN MEASURES}

For $\alpha \geqslant \beta \geqslant-1 / 2$ we consider the Jacobi polynomials

$$
P_{n}^{(\alpha, \beta)}(x):=\frac{1}{\left(\begin{array}{c}
n+\alpha \\
n
\end{array}\right) 2^{n}} \sum_{j=0}^{n}\left(\begin{array}{c}
n+\alpha \\
j
\end{array}\right)\left(\begin{array}{c}
n+\beta \\
n-j
\end{array}\right)(x-1)^{n-j}(x+1)^{j}, \quad x \in \mathbb{R}, n \in \mathbb{N}_{0}
$$

which are orthogonal on $[-1,1]$ with respect to the weight $(1-x)^{\alpha}(1+x)^{\beta}$ and normalised by $P_{n}^{(\alpha, \beta)}(1)=1$. (We shall also use the notation $P_{n}$ if it cannot cause misunderstanding.) It was shown by Gasper $[5,6]$ that for all $s, t \in[0, \pi]$ there exists a (unique) probability measure $\varepsilon_{s} * \varepsilon_{t}$ on $[0, \pi]$ such that

$$
P_{n}^{(\alpha, \beta)}(\cos s) \cdot P_{n}^{(\alpha, \beta)}(\cos t)=\int_{0}^{\pi} P_{n}^{(\alpha, \beta)}(\cos u) d\left(\varepsilon_{s} * \varepsilon_{t}\right)(u), \quad n \in \mathbb{N}
$$

The convolution $\varepsilon_{s} * \varepsilon_{t}$ can be extended uniquely to a bilinear, commutative, associative and weakly continuous convolution * on the Banach space $M_{b}([0, \pi])$ of all (signed) Borel measures on $[0, \pi]$. Moreover, * establishes a commutative hypergroup structure on $K=[0, \pi]$. Its normalised Haar measure $\omega=\omega^{(\alpha, \beta)}$ is given by

$$
d \omega(\vartheta):=\frac{\Gamma(\alpha+\beta+2)}{\Gamma(\alpha+1) \Gamma(\beta+1) 2^{\alpha+\beta+1}}(\sin \vartheta)^{2 \alpha+1}(1+\cos \vartheta)^{\beta-\alpha} d \vartheta, \quad \vartheta \in[0, \pi]
$$

The dual space $\widehat{K}=\left\{P_{n}^{(\alpha, \beta)} \circ \cos : n \in \mathbb{N}_{0}\right\}$ can be identified with $\mathbb{N}_{0}$, and the Plancherel measure $\pi=\pi^{(\alpha, \beta)}$ on it is given by

$$
\pi(\{n\}):=h_{n}^{(\alpha, \beta)}:=\frac{(2 n+\alpha+\beta+1)(\alpha+\beta+1)_{n}(\alpha+1)_{n}}{(\alpha+\beta+1) n !(\beta+1)_{n}}, \quad n \in \mathbb{N}_{0},
$$


where for $\gamma \in \mathbb{R}$ and $j \in \mathbb{N}$ we use the standard notation

$$
(\gamma)_{j}:=\gamma(\gamma+1) \cdots(\gamma+j-1)
$$

The Jacobi-Fourier transform of a measure $\mu \in M_{b}([0, \pi])$ and a function $f \in$ $L^{1}([0, \pi], \omega)$ are given by

$$
\widehat{\mu}(n):=\int_{0}^{\pi} P_{n}^{(\alpha, \beta)}(\cos \vartheta) d \mu(\vartheta), \quad \widehat{f}(n):=\int_{0}^{\pi} P_{n}^{(\alpha, \beta)}(\cos \vartheta) f(\theta) d \omega(\vartheta), \quad n \in \mathbb{N}_{0}
$$

According to the inversion theorem (see [3, Theorem 2.2.36]), if $\mu \in M_{b}([0, \pi])$ is such that

$$
\|\widehat{\mu}\|_{1}:=\sum_{n=0}^{\infty} h_{n}^{(\alpha, \beta)}|\widehat{\mu}(n)|<\infty
$$

then $\mu$ has a continuous $\omega$-density $f$ given by

$$
f(\vartheta):=\sum_{n=0}^{\infty} h_{n}^{(\alpha, \beta)} \widehat{\mu}(n) P_{n}^{(\alpha, \beta)}(\cos \vartheta), \quad \vartheta \in[0, \pi]
$$

and

$$
\|\mu\| \leqslant\|f\|_{\infty} \leqslant\|\hat{f}\|_{1}=\|\hat{\mu}\|_{1} .
$$

For all $\alpha \geqslant \beta \geqslant-1 / 2$ and $j \in \mathbb{N}$ we introduce the functions

$$
q_{j}(n):=q_{j}^{(\alpha, \beta)}(n):=\frac{n !(n+\alpha+\beta+1)_{j}}{2^{j}(n-j) !(\alpha+1)_{j}}, \quad n \in \mathbb{N}_{0} .
$$

In fact, $q_{j}$ is a polynomial of degree $2 j$ and in particular

$$
q_{1}(n)=\frac{n(n+\alpha+\beta+1)}{2(\alpha+1)}
$$

For $\sigma^{2}>0$ the Gaussian measure $\gamma_{\sigma^{2}}$ with parameter $\sigma^{2}$ on the Jacobi hypergroup can be defined by its Jacobi-Fourier transform

$$
\widehat{\gamma}_{\sigma^{2}}(n):=\exp \left\{-\frac{\sigma^{2} q_{1}(n)}{2}\right\}, \quad n \in \mathbb{N}_{0},
$$

see, for instance, Bochner [4]. Clearly $\left\|\widehat{\gamma}_{\sigma^{2}}\right\|_{1}<\infty$ implies that it has a continuous, bounded $\omega$-density. 


\section{EDGEWORTH EXPANSION}

Let $\mu$ be a probability measure on $[0, \pi]$. We denote the moments of $\mu$ by

$$
m_{j}:=m_{j}^{\mu}:=\int_{0}^{\pi} t^{j} d \mu(t), \quad j \in \mathbb{N}
$$

Let $\left(X_{n}\right)_{n \geqslant 0}$ be the Markov chain on $[0, \pi]$ associated with $\mu$ and on the Jacobi hypergroup as follows. The chain starts at 0 at time 0 , and the transition probabilities satisfy

$$
P\left(X_{n} \in A \mid X_{n-1}=z\right)=\left(\varepsilon_{z} * \mu\right)(A)
$$

for all $n \in \mathbb{N}, z \in[0, \pi]$ and Borel sets $A \subset[0, \pi]$. As in Voit [9] we obtain that the distribution of $X_{n}$ is given by the $n$-fold Jacobi convolution power $\mu^{(n)}$.

For all $k \in \mathbb{N}$ let $\mu_{k}$ be the probability measure on $[0, \pi / \sqrt{k}]$ defined by $\mu_{k}(A):=$ $\mu(\sqrt{k} A)$ for Borel sets $A \subset[0, \pi / \sqrt{k}]$. Let $\left(X_{n}^{(k)}\right)_{n \geqslant 0}$ be the Markov chain on $[0, \pi]$ associated with $\mu_{k}$. Then $X_{k}^{(k)}$ has distribution $\mu_{k}^{(k)}$ with its Jacobi-Fourier transform

$$
\widehat{\mu_{k}^{(k)}}(n)=\left(\widehat{\mu}_{k}(n)\right)^{k}=\left(\int_{0}^{\pi} P_{n}(\cos \theta) d \mu_{k}(\theta)\right)^{k}=\left(\int_{0}^{\pi} P_{n}(\cos (t / \sqrt{k})) d \mu(t)\right)^{k}
$$

The Edgeworth polynomials $Q_{j}^{\mu}, j \in \mathbb{N}_{0}$, corresponding to the measure $\mu$ are defined by the formal expansion

$$
\left(\int_{0}^{\pi} P_{n}(\cos (t / \sqrt{k})) d \mu(t)\right)^{k}=\exp \left\{-\frac{m_{2} q_{1}(n)}{2}\right\} \sum_{j=0}^{\infty} Q_{j}^{\mu}(n) k^{-j}
$$

More precisely, defining first the polynomials $\psi_{j}^{\mu}, j \in \mathbb{N}$, by the power series (in the variable $y$ )

$$
\frac{1}{2} m_{2} q_{1}(n)+\frac{1}{y^{2}} \log \int_{0}^{\pi} P_{n}^{(\alpha, \beta)}(\cos (t y)) d \mu(t)=\sum_{j=1}^{\infty} \psi_{j}^{\mu}(n) y^{2 j}
$$

we can determine the polynomials $Q_{j}^{\mu}, j \in \mathbb{N}_{0}$, by

$$
\exp \left\{\sum_{j=1}^{\infty} \psi_{j}^{\mu}(n) x^{j}\right\}=\sum_{j=0}^{\infty} Q_{j}^{\mu}(n) x^{j}
$$

(In Section 4 we prove that the above power series has a positive radius of convergence.) We remark that the Edgeworth polynomials $Q_{j}^{\mu}, j \in \mathbb{N}_{0}$, also depend on the parameters $\alpha$ and $\beta$ through the functions $q_{j}=q_{j}^{(\alpha, \beta)}, j \in \mathbb{N}$. The degree of the polynomial $Q_{j}^{\mu}$ 
is at most $2 j+2$, and the coefficients are polynomials of the moments $m_{2}, m_{4}, \ldots$, $m_{2(j+1)}$. In particular,

$$
\begin{gathered}
Q_{0}^{\mu}(n) \equiv 1, \quad Q_{1}^{\mu}(n)=m_{4}\left(\frac{q_{1}(n)}{24}+\frac{q_{2}(n)}{8}\right)-m_{2}^{2} \frac{\left(q_{1}(n)\right)^{2}}{8} \\
Q_{2}^{\mu}(n)=m_{2} m_{4}\left(\frac{\left(q_{1}(n)\right)^{2}}{48}+\frac{q_{1}(n) q_{2}(n)}{9}\right)-m_{2}^{3} \frac{\left(q_{1}(n)\right)^{3}}{24} \\
-m_{6}\left(\frac{q_{1}(n)}{720}+\frac{q_{2}(n)}{48}+\frac{q_{3}(n)}{48}\right)+\frac{1}{2}\left(Q_{1}^{\mu}(n)\right)^{2} .
\end{gathered}
$$

(The definition of the $Q_{j}^{\mu}, j \in \mathbb{N}_{0}$, should be compared with the usual expansion

$$
(\widehat{\mu}(t / \sqrt{k}))^{k}=\exp \left\{-\frac{1}{2} \sigma^{2} t^{2}\right\} \sum_{j=0}^{\infty} k^{-j / 2} \widetilde{P}_{j}^{\mu}(i t)
$$

of the characteristic function of a normalised convolution power of a probability measure $\mu$ on $\mathbb{R}$ with zero mean and covariance $\sigma^{2}$; see Bhattacharya and Ranga Rao [1, Section 7].) Furthermore, for all $j \in \mathbb{N}_{0}$ we define the signed measure $\nu_{j}^{\mu}$ on $[0, \pi]$ by its JacobiFourier transform

$$
\widehat{\nu_{j}^{\mu}}(n):=Q_{j}^{\mu}(n) \exp \left\{-\frac{m_{2} q_{1}(n)}{2}\right\}, \quad n \in \mathbb{N}_{0} .
$$

Clearly $\left\|\widehat{\nu}_{j}^{\mu}\right\|_{1}<\infty$, and hence $\nu_{j}^{\mu}$ exists and has a continuous, bounded $\omega$-density $g_{j}$. Obviously $\nu_{0}^{\mu}$ is just the Gaussian measure $\gamma_{\sigma^{2}}$ with parameter $\sigma^{2}=m_{2}$.

We need the following mild restriction on $\mu$, which means that ' $\mu$ is not concentrated in 0 too much'.

Condition (G). We say that a probability measure $\mu$ on $[0, \pi]$ has the growth property (G) at the point 0 if there exist constants $c, p>0$ such that

$$
\mu([0, \delta]) \leqslant c \cdot \delta^{p} \quad \text { for all } \delta \in[0, \pi] .
$$

Condition $(G)$ is equivalent to

$$
\int_{0}^{\pi} t^{-q} d \mu(t)<\infty \quad \text { for some } q>0
$$

see Voit [9, Remark 1.9].

From Voit [9, Theorem 1.6] if $\alpha \geqslant \beta \geqslant-1 / 2, \alpha>-1 / 2$, and $\mu$ is a probability measure on $[0, \pi]$ with property $(G)$ then there is a $k_{0}=k_{0}(d, \mu)$ such that for all $k \geqslant k_{0}$ the distribution $\mu_{k}^{(k)}$ of $X_{k}^{(k)}$ has a continuous, bounded $\omega$-density $f_{k}$. 
ThEOREM. Let $\alpha \geqslant \beta \geqslant-1 / 2, \alpha>-1 / 2$, and let $\mu$ be a probability measure on $[0, \pi]$ with property $(G)$. Then for all $s \in \mathbb{N}$,

$$
\left\|f_{k}-\sum_{j=0}^{s-1} k^{-j} \cdot g_{j}\right\|_{\infty}=O\left(k^{-s}\right) \quad \text { for } k \rightarrow \infty .
$$

In particular,

$$
\left\|\mu_{k}^{(k)}-\sum_{j=0}^{s-1} k^{-j} \cdot \nu_{j}^{\mu}\right\|=O\left(k^{-s}\right) \quad \text { for } k \rightarrow \infty
$$

\section{PRoOF OF THE THEOREM}

The letter $c$ with or without indices will denote a positive constant depending only on the fixed parameters $\alpha, \beta$ and the measure $\mu$. The same symbol may stand for different constants. A relationship $a_{k}=O\left(b_{k}\right)$ between sequences $\left(a_{k}\right)$ and $\left(b_{k}\right)$ of real numbers means that there exists a constant $c$ (depending only on $\alpha, \beta$ and $\mu$ ) such that $\left|a_{k}\right| \leqslant c b_{k}$ for all $k \in \mathbb{N}$.

In view of the properties of the Jacobi-Fourier transform (see Section 2) it is sufficient to show that

$$
\left\|\widehat{\mu_{k}^{(k)}}-\sum_{j=0}^{s-1} k^{-j} \cdot \widehat{\nu_{j}^{\mu}}\right\|_{1}=O\left(k^{-s}\right)
$$

We have

$$
\begin{aligned}
\| \widehat{\mu_{k}^{(k)}} & -\sum_{j=0}^{s-1} k^{-j} \cdot \widehat{\nu_{j}^{\mu}} \|_{1} \\
& =\sum_{n=0}^{\infty} h_{n}\left|\left(\int_{0}^{\pi} P_{n}(\cos (t / \sqrt{k})) d \mu(t)\right)^{k}-\exp \left\{-\frac{m_{2} q_{1}(n)}{2}\right\} \sum_{j=0}^{s-1} Q_{j}^{\mu}(n) k^{-j}\right| \\
& \leqslant R_{1}(k)+R_{2}(k)+R_{3}(k)
\end{aligned}
$$

where

$$
\begin{aligned}
& R_{1}(k):=\sum_{n \leqslant A \sqrt{k}} h_{n}\left|\left(\int_{0}^{\pi} P_{n}(\cos (t / \sqrt{k})) d \mu(t)\right)^{k}-\exp \left\{-\frac{m_{2} q_{1}(n)}{2}\right\} \sum_{j=0}^{s-1} Q_{j}^{\mu}(n) k^{-j}\right| \\
& R_{2}(k):=\sum_{n>A \sqrt{k}} h_{n}\left(\int_{0}^{\pi} P_{n}(\cos (t / \sqrt{k})) d \mu(t)\right)^{k} \\
& R_{3}(k):=\sum_{n>A \sqrt{k}} h_{n} \exp \left\{-\frac{m_{2} q_{1}(n)}{2}\right\} \sum_{j=0}^{s-1} Q_{j}^{\mu}(n) k^{-j}
\end{aligned}
$$


with arbitrary $A>0$, which will be chosen appropriately later.

Using the arguments leading to (3.10) in Voit [9] we observe that for each $A>0$ the term $R_{3}(k)$ tends exponentially to 0 as $k \rightarrow \infty$, that is,

$$
R_{3}(k) \leqslant c_{1} \cdot c_{2}^{k}
$$

for suitable constants $c_{1}>0$ and $0<c_{2}<1$.

By appealing to Voit [9, Lemma 3.8] if the measure $\mu$ has the property (G), then for each $A>0$ the term $R_{2}(k)$ also tends exponentially to 0 as $k \rightarrow \infty$ in the sense that

$$
R_{2}(k) \leqslant c_{1} \cdot c_{2}^{k} \quad \text { for all } k \geqslant c_{3}
$$

for suitable constants $c_{1}, c_{3}>0$ and $0<c_{2}<1$.

The aim of the following discussion is to show that for sufficiently small $A=$ $A(\mu, \alpha, \beta)$ we have

$$
R_{1}(k)=O\left(k^{-s}\right)
$$

In view of our normalisation $P_{n}^{(\alpha, \beta)}(1)=1$, Szegö $[8,(4.21 .7)$ and (4.1.1)] lead to the recursive formula

$$
\frac{d P_{n}^{(\alpha, \beta)}}{d x}(x)=\frac{n(n+\alpha+\beta+1)}{2(\alpha+1)} \cdot P_{n-1}^{(\alpha+1, \beta+1)}(x), \quad x \in \mathbb{R}, n \in \mathbb{N}
$$

Consequently we have

$$
\frac{d^{j} P_{n}^{(\alpha, \beta)}}{d x^{j}}(1)=q_{j}^{(\alpha, \beta)}(n), \quad j, n \in \mathbb{N},
$$

and we obtain easily the Taylor expansion for the Jacobi polynomials: there exists a constant $c$ such that

$$
\left|P_{n}(x)-\sum_{j=0}^{s} \frac{(-1)^{j}}{j !} q_{j}(n)(1-x)^{j}\right| \leqslant c n^{2(s+1)}(1-x)^{s+1}, \quad \text { for } x \in[-1,1], n \in \mathbb{N}_{0} .
$$

Using the Taylor expansion of the cosine function, we obtain

$$
\left|\int_{0}^{\pi} P_{n}(\cos (t y)) d \mu(t)-\sum_{j=0}^{s} \varphi_{j}(n) m_{2 j} y^{2 j}\right| \leqslant c n^{2(s+1)} y^{2(s+1)}
$$

for $y \in \mathbb{R}, n \in \mathbb{N}_{0}$ with some polynomials $\varphi_{j}, j \in \mathbb{N}_{0}$, where $\varphi_{0}(n)=1, \varphi_{1}(n)=$ $-q_{1}(n) / 2$ for $n \in \mathbb{N}_{0}$, and $\varphi_{j}$ is a polynomial of degree $2 j$. In particular, we can choose $A_{1}>0$ such that

$$
\left|\int_{0}^{\pi} P_{n}(\cos (t y)) d \mu(t)-1\right| \leqslant \frac{1}{2} \quad \text { for } y \in \mathbb{R} \text { and } n \in \mathbb{N}_{0} \text { with } n|y| \leqslant A_{1} .
$$


Applying the Taylor inequality

$$
\left|\log (1+x)-\sum_{j=1}^{s-1} \frac{(-1)^{j-1}}{j} x^{j}\right| \leqslant c|x|^{s}, \quad \text { for }|x| \leqslant \frac{1}{2}
$$

we conclude

$$
\left|\frac{1}{2} m_{2} q_{1}(n)+\frac{1}{y^{2}} \log \int_{0}^{\pi} P_{n}(\cos (t y)) d \mu(t)-\sum_{j=1}^{s-1} \psi_{j}^{\mu}(n) y^{2 j}\right| \leqslant C_{s} n^{2(s+1)} y^{2 s}
$$

for $y \in \mathbb{R}$ and $n \in \mathbb{N}_{0}$ with $n|y| \leqslant A_{1}$ and with some polynomials $\psi_{j}, j \in \mathbb{N}$, where $\psi_{j}$ is a polynomial of degree at most $2 j+2$. In particular, we have

$$
\left|\frac{1}{2} m_{2} q_{1}(n)+\frac{1}{y^{2}} \log \int_{0}^{\pi} P_{n}(\cos (t y)) d \mu(t)\right| \leqslant C_{2} n^{4} y^{2} \leqslant \frac{m_{2}}{8(\alpha+1)} n^{2}
$$

for $y \in \mathbb{R}$ and $n \in \mathbb{N}_{0}$ with $n|y| \leqslant A$, where

$$
A:=\min \left\{A_{1}, \sqrt{\frac{m_{2}}{8 C_{2}(\alpha+1)}}\right\} .
$$

With the help of the Taylor inequality

$$
\left|e^{x}-\sum_{j=0}^{s-1} \frac{x^{j}}{j !}\right| \leqslant \frac{|x|^{s}}{s !} e^{|x|}, \quad \text { for } x \in \mathbb{R}
$$

and putting $y=1 / \sqrt{k}$ we obtain

$$
\begin{gathered}
\left|\left(\int_{0}^{\pi} P_{n}(\cos (t / \sqrt{k})) d \mu(t)\right)^{k} \exp \left\{\frac{m_{2} q_{1}(n)}{2}\right\}-\sum_{j=0}^{s-1} Q_{j}^{\mu}(n) k^{-j}\right| \\
\leqslant c n^{4 s} k^{-s} \exp \left\{\frac{m_{2}}{8(\alpha+1)} n^{2}\right\}
\end{gathered}
$$

for $n \leqslant A \sqrt{k}$. Obviously

$$
q_{1}(n)=\frac{n(n+\alpha+\beta)}{2(\alpha+1)} \geqslant \frac{n^{2}}{2(\alpha+1)}
$$

implies

$$
\exp \left\{-\frac{m_{2} q_{1}(n)}{2}\right\} \leqslant \exp \left\{-\frac{m_{2}}{4(\alpha+1)} n^{2}\right\}
$$


and hence we have

$$
\begin{gathered}
\left|\left(\int_{0}^{\pi} P_{n}(\cos (t / \sqrt{k})) d \mu(t)\right)^{k}-\exp \left\{-\frac{m_{2} q_{1}}{2} \frac{(n)}{3-1}\right\} \sum_{j=0}^{s-1} Q_{j}^{\mu}(n) k^{-j}\right| \\
\leqslant c_{1} n^{4 s} k^{-s} \exp \left\{-\frac{m_{2}}{8(\alpha+1)} n^{! !}\right\}
\end{gathered}
$$

for $n \leqslant A \sqrt{k}$. Taking into account that

$$
h_{n}=O\left(n^{2 \alpha+1}\right),
$$

we conclude that

$$
R_{1}(k) \leqslant c_{1} \sum_{n \leqslant A \sqrt{k}} n^{2 \alpha+1} n^{4 s} k^{-s} e^{-c_{2} n^{2}}=O\left(k^{-s}\right) .
$$

\section{REFERENCES}

[1] R.N. Bhattacharya and R. Ranga Rao, Normal approximation and asymptotic expansions (John Wiley and Sons, New York, London, Sydney, Toronto, 1976).

[2] N.H. Bingham, 'Random walks on spheres', Z. Wahrscheinlichkeitsth. verw. Gebiele 22 (1972), 169-192.

[3] W.R. Bloom and H. Heyer, Harmonic analysis of probability measures on hypergroups (De Gruyter, Berlin, New York, 1995).

[4] S. Bochner, 'Positivity of the heat kernel for ultraspherical polynomials and similar functions', Arch. Rational Mech. Anal. 70 (1979), 211-217.

[5] G. Gasper, 'Positivity and convolution structure for Jacobi series', Ann. Math. 93 (1971), 112-118.

[6] G. Gasper, 'Banach algebras for Jacobi series and positivity of a kernel', Ann. Math. 95 (1972), 261-280.

[7] S. Helgason, Groups and geometric analysis (Academic Press, Orlando, Fl., 1984).

[8] G. Szegö, Orthogonal polynomials, Amer. Math. Soc. Coll. Publ. 23 (Amer. Math. Soc., Providence R.I., 1959).

[9] M. Voit, 'Rate of convergence to Gaussian measures on $\boldsymbol{n}$-spheres and Jacobi hypergroups', Ann. Probab. 25 (1997), 457-477.

Institute of Mathematics

Lajos Kossuth University of Debrecen

Egyetem tér 10

H-4010 Debrecen

Hungary

e-mail: papgy@math.klte.hu
Mathematisches Institut

Universität Tübingen

Auf der Morgenstelle 10

D-72076 Tübingen

Germany

e-mail: voit@uni-tuebingen.de 